\title{
Promise and Perils of Telehealth in the Current Era
}

\author{
Dhruv Mahtta $^{1,2} \cdot$ Marilyne Daher $^{3} \cdot$ Michelle T. Lee $^{1,4} \cdot$ Saleem Sayani $^{5} \cdot$ Mehdi Shishehbor $^{6} \cdot$ Salim S. Virani ${ }^{1,2,4}$ (I)
}

Accepted: 24 March 2021 / Published online: 16 July 2021

(C) This is a U.S. government work and not under copyright protection in the U.S.; foreign copyright protection may apply 2021

\begin{abstract}
Purpose of Review The concept of telehealth has been around since the early twentieth century and has been used in different healthcare specialties. However, with the recent COVID-19 pandemic necessitating physical distancing, there has been an increased emphasis and utilization of this mode of healthcare delivery. With increasing reliance on telehealth services, data from investigator groups have brought to light several merits as well as failings of telehealth.

Recent Findings Telehealth services have been associated with improved healthcare outcomes while remaining a cost-effective mode of healthcare delivery. Improving access and timeliness of care has also been observed by multiple telehealth-related studies. Finally, telehealth services are also anticipated to serve as part of emergency preparedness protocol and have shown to reduce provider-patient supply-demand mismatch, prevalent in certain subspecialties. With these benefits come certain challenges that have been highlighted in the literature. Indiscriminate utilization of telehealth services may widen public health disparities among minority groups and may increase overall healthcare expenditure due to overutilization of care, and the digital platform may jeopardize security of patient data.

Summary COVID-19 has been a catalyst in increasing utilization of telehealth services. As we move forward from the current pandemic, lessons learned from the studies demonstrating benefits and challenges associated with telehealth should be taken into account when drafting post-pandemic telehealth policies. Special attention should be paid to ensure that telehealth narrows, and not widens, the currently existing disparities in access to healthcare.
\end{abstract}

Keywords COVID-19 $\cdot$ Healthcare disparities $\cdot$ Overutilization $\cdot$ Telemedicine $\cdot$ Telehealth services

\section{Introduction}

Amidst the COVID-19 pandemic, telehealth has emerged as a rapidly expanding service connecting patients with their healthcare providers in an efficient, cost-effective, and physically distant manner [1-3]. The Centers of Medicare and Medicaid services (CMS) defines telehealth as "the use of telecommunications and information technology to provide

This article is part of the Topical Collection on Public Health Policy

Salim S. Virani

virani@bcm.edu

Dhruv Mahtta

dhruv.mahtta@bcm.edu

Marilyne Daher

marilyne.daher@bcm.edu

Michelle T. Lee

michelle.lee@bcm.edu

Saleem Sayani

saleem.sayani@aku.edu

Mehdi Shishehbor

mehdi.shishehbor@uhhospitals.org
1 Section of Cardiology, Michael E. DeBakey Veterans Affairs Medical Center, 2002 Holcombe Blvd, Houston, TX, USA

2 Section of Cardiology, Department of Medicine, Baylor College of Medicine, Houston, TX, USA

3 Department of Medicine, Baylor College of Medicine, Houston, TX, USA

4 Health Policy, Quality \& Informatics Program, Michael E. DeBakey VA Medical Center Health Services Research \& Development Center for Innovations in Quality, Effectiveness, and Safety, Houston, TX, USA

5 Aga Khan Development Network, eHealth Resource Center for South-Central Asia and East Africa, Karachi, Pakistan

6 Department of Cardiology, University Hospitals Harrington Heart and Vascular Institute, Cleveland, OH, USA 
access to health assessment, diagnosis, intervention, consultation, supervision, and information across distance" [4]. The delivery of telehealth can be synchronous, with clinicians and patients interacting in real time, or asynchronous, whereby data trends or messages are shared with clinicians in a periodic manner or can be utilized as remote patient monitoring. Although the concept of telehealth has existed since the early twentieth century, its implementation and integration have relatively lagged behind. Suboptimal payment structures and regulatory laws have often been criticized as reasons behind incomplete adoption of telehealth $[5,6]$.

In light of the current pandemic, there has been an increasing reliance on the telehealth platform to provide high-quality care. Within the last year, the landscape of telehealth has markedly evolved with newer legislation supporting seamless integration of telehealth and overcoming barriers surrounding compensation structure $[7,8]$. As clinical workflow via telehealth becomes the "new normal," it is imperative to review and have a deeper level of understanding of the key promises and perils of telehealth. In this contemporary review article, we discuss the role of telehealth in the current healthcare arena, its advantages, and challenges associated with its use. We also aim to highlight future implementation initiatives which are on the horizon.

\section{Changing Landscape of Telehealth}

The COVID-19 pandemic has served as a catalyst in advancing telehealth technology as well as increasing its utilization and integration into the current clinical environment. Single center metrics from the Oregon Health and Science University demonstrated a drastic increase from 1100 telehealth visits pre-COVID-19 pandemic to 13,000 during the pandemic [9]. Similar trends have been observed across the nation with multi-center analyses demonstrating 50 to $154 \%$ increase in telehealth visits for both COVID-19- and non-COVID-19related encounters between 2019 and 2020 [10•]. Data from China, France, Switzerland, and Brazil have also confirmed similar rise in utilization of telehealth $[11,12]$. Legislations such as the US Coronavirus Aid, Relief, and Economic Security (CARES) Act and regulatory waivers passed by the Centers for Medicare and Medicaid Services (CMS) have catapulted telemedicine from being restricted to certain fields of medicine to all medical, mental health, pediatric, and surgical sub-specialties [13••, 14-16]. Finally, a systematic review comparing videoconferencing and teleconferencing interviews demonstrated that although videoconferencing took longer, providing telehealth services via videoconferencing was associated with more accurate diagnoses, efficient treatment decisions, and fewer medication errors as compared to teleconferencing. There were no significant differences in patient satisfaction and mortality based on care provided via either modality [17].

\section{Benefits of Telehealth}

Multiple investigations prior to the COVID-19 pandemic have demonstrated benefits of telehealth services. Pertaining to the cardiovascular community, a 2017 meta-analysis demonstrated that heart failure patients managed via telemedicine in addition to the standard-of-care in-person visits were observed to have lower all-cause mortality, lower heart failure admission rates, and shorter admission length of stay compared to patients managed via in-person only approach [18]. This was partially attributed to earlier recognition of symptoms and thus earlier interventions and guided self-management. Similarly, in the primary care arena, a Cochrane systematic review showed that glycated hemoglobin levels (HbAlc) were lower in the telemedicine group compared to those in standard of care [19]. These clinically driven benefits of telehealth prior to, during, and even after the pandemic may be attributed to several factors including improved cost-effectiveness, access and timeliness of care, emergency preparedness, and providerpatient mismatch reduction in certain sub-specialties.

\section{Cost-effectiveness}

The cost-effectiveness of telehealth services has been evaluated and demonstrated by prior observational studies $[2,20]$. From the patient's perspective, telehealth services offer costsavings via cutting down on travel time, extended time off work, and costs associated with emergency department and clinic visits [20]. Based on their observational study, Nord et al. demonstrated that triaging patients appropriately and thereby avoiding unnecessary emergency department visits yielded \$19-121 net savings per telehealth visit [21]. Focusing directly on travel costs, a 17 -year-long Californiabased study computed a savings of $\$ 2.8$ million dollars per annum in travel costs averted by telehealth services [22]. The cost-effectiveness of telehealth is not limited to patients but also extends to the healthcare system as a whole. Appropriate triaging via telehealth platforms allows for savings via preventing misuse of healthcare resources. Prediction models have suggested that a reduction in emergency department visits by $1 \%$ via telehealth services may yield a net savings of over \$100 million per year [23]. Texas Department of Clinical Justice's telemedicine system demonstrated a decrease in emergency department visits up to $85 \%$ which equated to approximate savings of $\$ 780$ million over 14 years [24]. In light of this data, continued improved and integration of telehealth services in the current healthcare arena may prove cost-effective from the standpoint of patients as well as healthcare resources. 


\section{Improving Access and Timeliness of Care}

Telehealth interventions have shown to be promising against the challenges of access and timeliness of care both in the primary care and sub-specialty settings. Based on a large survey of adults living in rural areas, greater than one-fourth of adults reported difficulty accessing healthcare in the last year, with healthcare costs and distance being the most cited reasons [25]. Challenges in travelling to nearest hospital and their PCP were cited by $27 \%$ and $23 \%$ of the survey respondents, respectively [26]. Telehealth services have been shown to bridge this gap in access to care. Zhang and colleagues shared their "telestroke" program which was comprised of videoconferencing between physicians in underserved facilities and neurovascular specialists in large urban hospitals capable of providing guidance in treatment and remote triage as well as initiate management in patients with suspected strokes. The authors demonstrated that their "telestroke" program was associated with a higher likelihood of providing life-saving treatment (i.e., intravenous tissue plasminogen activator) and mechanical thrombectomy in a timely manner as compared to standard (in-person) of care [26]. As part of telehealth, "telementoring" programs such as project ECHO (Extension for Community Healthcare Outcomes) have emerged and equipped clinicians working in rural areas to connect with experts from different subspecialties for guidance and consultation regarding high-quality contemporary healthcare practices [27]. Improving access to quality healthcare has been an evolving public health challenge which may be partially solved by telehealth services.

\section{Improving Emergency Preparedness}

Lessons learned during the recent COVID-19 pandemic and associated utilization of telehealth services poses the question whether telehealth should be incorporated as an essential tool within emergency preparedness protocols [28]. During public health emergencies, surges in healthcare resources and staff demand can be met by clinicians from unaffected areas via the telehealth platform. A set plan for communication, coordination, and reimbursement of telehealth services during such times of need may be drafted into local emergency preparedness protocols. The value of telehealth services during such scenario was demonstrated via "Virtual First Responders" during hurricanes Harvey and Irma whereby a greater than twothirds of telehealth users were noted to be first-time user [29]. Temporary policies put in place to quickly mobilize telehealth resources can prove to be invaluable during times of public health calamities.
Reducing Provider-Patient Supply-Demand Mismatch

Among specialties experiencing staff shortages due to increased demand or decreased supply (i.e., clinician retirement), telehealth services can prove to be extremely valuable resource. The supply-demand mismatch can be mitigated by utilizing existing providers to work more hours from the comfort of their home and also encourage semi-retired clinicians to use telehealth services to meet unmet needs of a certain subspecialty. One such initiative that has been studied has been the MAVEN project [30] which promises to address physician shortages in underserved areas of growing shortages by recruiting semi-retired physician volunteers. Although not a perfect solution, telehealth platform promises to bridge the widening supply-demand gap in underserved communities.

\section{Challenges of Telehealth}

Although telehealth services seem beneficial in multiple scenarios and provide a promising solution to many public health obstacles, the use of these services is not without certain challenges. As telehealth utilization becomes more prevalent, especially during the ongoing pandemic, the challenges associated with the widespread use of these services have become more apparent. Some of the key challenges include overutilization or misuse of care, widening disparities in care, and patient data security. Without addressing these challenges in a thorough fashion, telehealth practices may prove to be counterproductive to the mission of advancing public health.

\section{Overutilization or Misuse of Care}

With the convenience of telehealth services comes the risk of unintentional use of these services for reasons which may not have otherwise triggered an in-person visit. Although access to care may be improved with telehealth services, this could worsen the already high healthcare cost burden. Estimates from the Congressional Budget Office have predicted an addition of 150,000 tele-visits in the year 2021 with an exponential growth through 2029 accounting for an estimated \$1 billion increase in healthcare expenditure between 2021 and 2029 [31]. Although the concept behind telehealth visit is to replace in-person visits, 300,000 patient analysis by Ashwood et al. demonstrated that approximately $90 \%$ of telehealth visits are constituted of additional visits rather than utilization in place of in-person visits [32]. With advancement in technology and increasing utilization of digital platforms worldwide, misuse of healthcare resources via telehealth platforms remains a real issue. Policies surrounding the use of telehealth during non-pandemic times should provide careful consideration towards equality, and checkpoints be put into place to avoid inadvertent overutilization of healthcare resources 
which may drive up the overall healthcare expenditure without a net public health benefit.

\section{Widening Disparities in Care}

While there is risk of populations in urban areas equipped with latest generation technology overutilizing telehealth services, certain rural or underserved patient populations may see a widening inequality in access to healthcare via a telehealth platform. One of the key requirements for a successful telehealth visit, particularly when it involves video visits, is access to high-speed (broadband) Internet. According to the Federal Communication Commissions, 33\% of the rural population lacks broadband Internet access [33]. This increases disparities in care among the rural and already underserved population by further increasing the inequalities in accessing healthcare. Similar barriers to accessing telehealth services may also prevail among racial minority groups such as Blacks and Hispanic population, those who are not fluent in English language, and those with limited digital literacy [34•]. Disparities in access to broadband subscription and hence access to telehealth services were evident prior to the COVID-19 pandemic, but the increasing reliance of these services during the pandemic has widened these disparities. Recent studies consisting of patients with diabetes mellitus and atherosclerotic cardiovascular disease have demonstrated that racial minorities such as Blacks and Hispanics are less likely to report Internet usage as compared to their White counterparts [35]. This disparity is also associated with significant statewide variation. Based on their nationwide survey, the authors demonstrated that these patients who require more healthcare resources given their baseline comorbid condition were $26 \%$ less likely to have Internet access [36]. Based on data from the New York health system, although the population consisted of $42 \%$ White, 23\% Black, 14\% Asian, and 7\% Hispanic patients, users of telehealth only consisted of $17 \%$ Black, $8 \%$ Asian, and 5\% Hispanic population, while greater than $50 \%$ of users were White [34•]. In another study evaluating virtual visits with otolaryngologists, Black patients, those with Medicaid insurance, and those in the third and lowest quartile of median household income census blocks were less likely to utilize telehealth services [37]. Such data highlight the need to address these racial and socioeconomic disparities pertaining to broadband access and access to telehealth services in order to ensure that telehealth is used as a tool to narrow rather than widen gap in healthcare access.

\section{Patient Data Security}

During the COVID-19 pandemic, legislations allowing for non-HIPAA-compliant video platforms raised concerns that a substantial portion of these online communications lacked end-to-end encryption [38], leaving patient data vulnerable to interpretation upon interception. Even before the current leniency in legislations, concerns have circulated regarding HIPAA compliance of numerous telehealth platforms and potential compromise of patient data. Investigators evaluating 600 mobile health applications demonstrated that approximately $30 \%$ of these applications had a privacy policy in place [39]. A lack of privacy policy may lead to inadvertent vulnerability and breach of patient health information. Furthermore, different telehealth avenues of sharing information and remotely transmitting device data have also put manufacturers of these devices under scrutiny as such devices are themselves at risk for jeopardizing patients' protected health information [40]. As telehealth becomes more prevalent in the healthcare arena, it is important for policymakers to ensure that easy-tounderstand privacy policies are in place for patients and clinicians to understand the security of respective telehealth platforms, in addition to having regulatory policies for telehealth applications and platforms to mitigate security risks.

\section{Future Directions and Conclusions}

Telehealth services have reemerged and gained attention during the ongoing COVID-19 pandemic with investigations evaluating promises and perils of telehealth in the current era (Fig. 1). As the healthcare community moves forward from the current pandemic, it will be important to note whether the temporary pandemic-related expansion in telehealth policies remains into effect or is reverted to pre-pandemic policies. The marked increase in telehealth utilization has brought to light several distinct advantages while also highlighting key shortcomings of telehealth. It is imperative for policymakers to consider the current benefits and impediments of telehealth prior to articulating post-pandemic goals of telehealth services. A key focus of postpandemic policies regarding telehealth utilization should revolve around narrowing the inequalities in access to care and increase access for minority groups. Careful consideration is also needed to carve post-pandemic policies to derive maximum benefit from telehealth without inflating the current healthcare expenditure. Utilization management strategies such as lifting restrictions from underserved community, reimbursements for telephone-only visits for rural communities without broadband, and imposing frequency limitations on the number of telehealth visits for urban population are some ways to ensure that telehealth services are directed in areas of increased need. Telehealth remains an essential part of the current healthcare infrastructure but, however, requires conscientious policy drafting to ensure that the healthcare community yields its intended benefits without widening disparities in care or misusing resources. Finally, healthcare community would also need to remain vigilant regarding adequate quality control and supervision as the field of telehealth continues to expand. With escalating demand for healthcare during these trying times and 


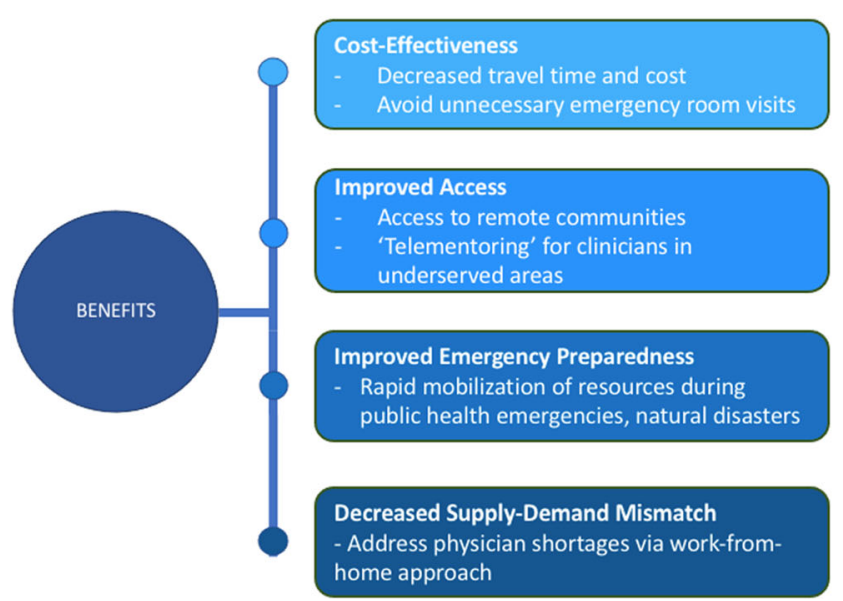

Fig. 1 Benefits and challenges associated with telehealth services

demands being met with expanding telehealth service, it is important to outline clear guidelines to ensure streamlined care and high-quality care as that of in-person visits.

\section{Declarations Conflict of Interest}

Dr. Virani reports research support: Department of Veterans Affairs, World Heart Federation, Tahir and Jooma Family, and Honorarium American College of Cardiology (Associate Editor for Innovations, aacc.org). Dr. Shishehbor serves on the global advisory board for Medtronic, Abbott Vascular, Terumo Medical Corporation, Philips, and Boston Scientific. The other authors declare that they have no conflict of interest.

Human and Animal Rights and Informed Consent This article does not contain any studies with human or animal subjects performed by any of the authors.

\section{References}

Papers of particular interest, published recently, have been highlighted as:

- Of importance

•- Of major importance

1. Calton B, Abedini N, Fratkin M. Telemedicine in the time of coronavirus. J Pain Symptom Manag. 2020;60(1):e12-4.

2. Greenhalgh T, Wherton J, Shaw S, et al. Video consultations for covid-19. BMJ. 2020;368:m998.

3. Ducharme J The coronavirus outbreak could finally make telemedicine mainstream in the U.S. TIME: Time Magazine, 2020.

4. Hanson KW, Huskamp HA. Behavioral health services under Medicaid managed care: the uncertain implications of state variation. Psychiatric Serv (Washington, DC). 2001;52(4):447-50.

5. Keesara S, Jonas A, Schulman K. Covid-19 and health care's digital revolution. N Engl J Med. 2020 Jun 4;382(23):e82.

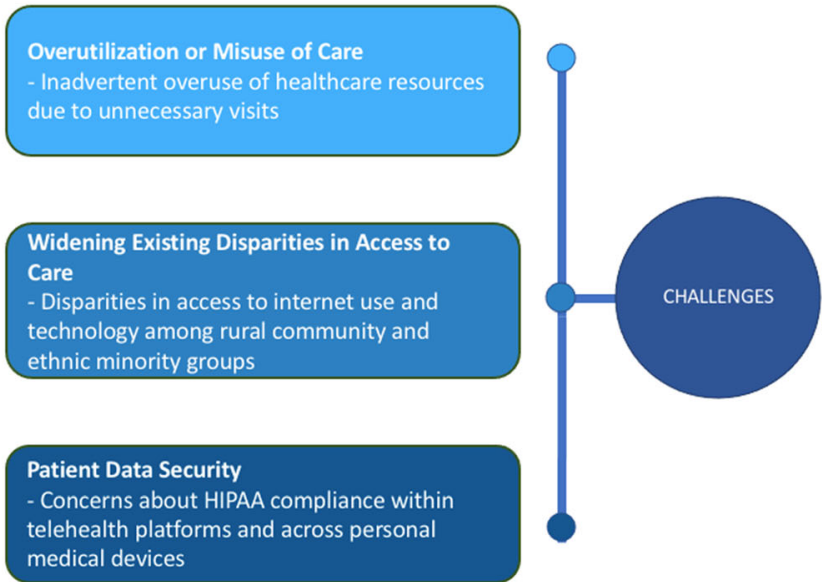

6. Scott Kruse C, Karem P, Shifflett K, Vegi L, Ravi K, Brooks M. Evaluating barriers to adopting telemedicine worldwide: a systematic review. J Telemed Telecare. 2018;24(1):4-12.

7. Kichloo A, Albosta M, Dettloff K, Wani F, El-Amir Z, Singh J, et al. Telemedicine, the current COVID-19 pandemic and the future: a narrative review and perspectives moving forward in the USA. Family Med Community Health. 2020;8(3):e000530.

8. Schmit C, Schwitzer J, Survance K, Barbre M, Nmadu Y, McCord C, et al. Assessing legal responses to COVID-19. Boston: Public Health Law Watch; 2020.

9. Robinson E OHSU telehealth rockets into 'new era of medicine': global pandemic instigates exponential expansion of OHSU telemedicine program OHSU News 2020 [Available from: https:// news.ohsu.edu/2020/04/13/ohsu-telehealth-rockets-into-new-eraof-medicine.] Accessed $20 \mathrm{Feb} 2021$.

10. - Koonin LM, Hoots B, Tsang CA, Leroy Z, Farris K, Jolly B, Antall P, McCabe B, Zelis CB, Tong I, Harris AM. Trends in the use of telehealth during the emergence of the COVID-19 pandemic - United States, January-March 2020. Morb Mortal Wkly Rep 2020;69(43):1595. Report highlighting trends in utilization of telehealth services with emergences of the COVID-19 pandemic.

11. Ohannessian R, Yaghobian S. The practicality of telemedicine and telehealth during the COVID-19 global outbreak. Eur J Pub Health. 2020;30(Supplement 5):ckaa165-069.

12. Caetano R, Silva AB, Guedes AC, Paiva CC, Ribeiro GD, Santos DL, et al. Challenges and opportunities for telehealth during the COVID-19 pandemic: ideas on spaces and initiatives in the Brazilian context. Cadernos de Saúde Pública. 2020;36:e00088920.

13.. . CARES Act: AMA COVID-19 pandemic telehealth fact sheet. 2020. Available at: https://www.ama-assn.org/delivering-care/ public-health/cares-act-ama-covid-19- pandemic-telehealth-factsheet. Accessed February 26, 2021. Document highlighting the nuances of the recent legislation approved to facilitate expansion of telehealth services.

14. Centers for Medicare \& Medicaid Services (CMS), 2020; Health Resources and Service Administration, 2020; and Pub. L. 116-136, 2020.

15. Wang CJ, Car J, Zuckerman BS. The power of telehealth has been unleashed. Pediatr Clin. 2020;67(4):xvii-xviii.

16. Parisien RL, Shin M, Constant M, Saltzman BM, Li X, Levine WN, et al. Telehealth utilization in response to the novel coronavirus 
(COVID-19) pandemic in orthopaedic surgery. J Am Acad Orthopaedic Surg. 2020;28(11):e487-92.

17. Rush KL, Howlett L, Munro A, Burton L. Videoconference compared to telephone in healthcare delivery: a systematic review. Int J Med Inform. 2018;118:44-53.

18. Lin MH, Yuan WL, Huang TC, Zhang HF, Mai JT, Wang JF. Clinical effectiveness of telemedicine for chronic heart failure: a systematic review and meta-analysis. J Investig Med. 2017;65(5): 899-911.

19. Flodgren G, Rachas A, Farmer AJ, Inzitari M, Shepperd S. Interactive telemedicine: effects on professional practice and health care outcomes. Cochrane Database Syst Rev. 2015(9):CD002098.

20. Powell RE, Henstenburg JM, Cooper G, Hollander JE, Rising KL. Patient perceptions of telehealth primary care video visits. Ann Fam Med. 2017;15(3):225-9.

21. Nord G, Rising KL, Band RA, Carr BG, Hollander JE. On-demand synchronous audio video telemedicine visits are cost effective. Am J Emerg Med. 2019;37(5):890-4.

22. Dullet NW, Geraghty EM, Kaufman T, Kissee JL, King J, Dharmar $\mathrm{M}$, et al. Impact of a university-based outpatient telemedicine program on time savings, Travel Costs, and Environmental Pollutants. Value Health. 2017;20(4):542-6.

23. Telehealth satisfaction study: J.D. power. 2019.

24. Smith DSG, Lewis B. Why telemedicine, why now? 2019 [Available from: https://www.americantelemed.org/resources/ why-telemedicine-why-now/. Accessed 20 Feb 2021.

25. Life in rural America Part II. NPr, the Robert Wood Johnson Foundation, and the Harvard TH Chan School of Public Health.

26. Zhang D, Wang G, Zhu W, Thapa JR, Switzer JA, Hess DC, et al. Expansion of telestroke services improves quality of care provided in super rural areas. Health Aff (Millwood). 2018;37(12):2005-13.

27. Arora S, Kalishman SG, Thornton KA, Komaromy MS, Katzman JG, Struminger BB, et al. Project ECHO: a telementoring network model for continuing professional development. J Contin Educ Health Prof. 2017;37(4):239-44.

28. Abir M, Nelson CD, Chan EW, Al Ibrahim H, Cutter C, Patel K, Bogart A. Critical care surge response strategies for the 2020 COVID-19 outbreak in the United States. RAND 2020.

29. Uscher-Pines L, Fischer S, Tong I, Mehrotra A, Malsberger R, Ray $\mathrm{K}$. Virtual first responders: the role of direct-to-consumer telemedicine in caring for people impacted by natural disasters. J Gen Intern Med. 2018 Aug;33(8):1242-4.
30. Uscher-Pines L, Rudin RS. Lessons learned from the MAVEN project pilot: using physician volunteers to increase access to care via telehealth. Rand Health Q. 2020;9(1):9.

31. U.S. Congress, House of Representatives, Beneficiary Education Tools, Telehealth, and Extenders Reauthorization Act of 2019 , H.R. 3417, 116th Congress, bill introduced in the House, 2019.

32. Ashwood JS, Mehrotra A, Cowling D, UscherPines L. Direct-toconsumer telehealth may increase access to care but does not decrease spending. Health Aff. 2017;36(3):485-91.

33. Drake C, Zhang Y, Chaiyachati KH, Polsky D. The limitations of poor broadband internet access for telemedicine use in rural America: an observational study. Ann Intern Med. 2019;171(5): $382-4$.

34. - Julien HM, Eberly LA, Adusumalli S. Telemedicine and the forgotten America. Circulation. 2020;142(4):312-4 Contemporary commentary on barriers with access to telehealth services.

35. Jain V, Al Rifai M, Lee MT, Kalra A, Petersen LA, Vaughan EM, et al. Racial and geographic disparities in Internet use in the US among patients with hypertension or diabetes: implications for telehealth in the era of COVID-19. Diabetes Care. 2021;44(1): e15-7.

36. Al Rifai M, Shapiro MD, Sayani S, Gulati M, Levine G, Rodriguez $\mathrm{F}$, et al. Racial and geographic disparities in Internet use in the United States among patients with atherosclerotic cardiovascular disease. Am J Cardiol. 2020;134:146-7.

37. Darrat I, Tam S, Boulis M, Williams AM. Socioeconomic disparities in patient use of telehealth during the coronavirus disease 2019 surge. JAMA Otolaryngol Head Neck Surg. 2021;147:287-95.

38. Fang J, Liu YT, Lee EY, Yadav K. Telehealth solutions for inhospital communication with patients under isolation during COVID-19. West J Emerg Med. 2020;21(4):801-6.

39. Sunyaev A, Dehling T, Taylor PL, Mandl KD. Availability and quality of mobile health app privacy policies. J Am Med Inform Assoc. 2015;22(e1):e28-33.

40. Seh AH, Zarour M, Alenezi M, Sarkar AK, Agrawal A, Kumar R, et al. Healthcare data breaches: insights and implications. Healthcare (Basel). 2020;8(2):133.

Publisher's Note Springer Nature remains neutral with regard to jurisdictional claims in published maps and institutional affiliations. 